\title{
Effect of the electronic subsystem excitation on the ionisation probability of atoms sputtered from metals by atomic and molecular projectiles
}

\author{
S.F. Belykh ${ }^{\mathrm{a}, *}$, I.A. Wojciechowski ${ }^{\mathrm{b}}$, V.V. Palitsin ${ }^{\mathrm{a}}$, A.V. Zinoviev ${ }^{\mathrm{c}}$, \\ A. Adriaens ${ }^{\text {a }}$, F. Adams ${ }^{\text {a }}$ \\ a Department of Chemistry, University of Antwerp (UIA), B-2610 Antwerp (Wilrijk), Belgium \\ ${ }^{\mathrm{b}}$ Université Catholique de Louvain, Place Croix du Sud 1, PCPM, 1348 Louvain-la-Neuve, Belgium \\ c NPO Akadempribor, 700143 Tashkent, Uzbekistan
}

Received 10 March 2001; accepted for publication 17 April 2001

\begin{abstract}
In the present work an effect of excitation of the metal electronic subsystem on the ionisation probability of atoms sputtered under fast ion bombardment has been studied. Atomic and molecular primary ions with the same velocity were used to produce different degrees of the electronic excitations. Information on the ionisation probability was obtained from the kinetic energy distributions of $\mathrm{Nb}^{+}$and $\mathrm{Ta}^{+}$ions sputtered from the respective clean $\mathrm{Nb}$ and $\mathrm{Ta}$ targets by $\mathrm{Au}_{m}^{-}$projectiles $(1 \leqslant m \leqslant 3)$ with the energy of $E_{0}=6 \mathrm{keV}$ per atom. It was found that, as compared with the atomic ion bombardment $(m=1)$, the molecular one $(m=2,3)$ leads to the increase of the ionisation probability $P_{m}^{+}$ $\left(P_{1}^{+}<P_{2}^{+}<P_{3}^{+}\right)$. Such an effect depends on the kinetic energy $E$ of the secondary ions, increasing with decreasing $E$. It was shown that the bombardment of metals by the molecular projectiles produces non-additive sputtering of atomic ions, which is determined by the joint action of such two factors as non-additive sputtering of atoms and non-additive process of their charge state formation. The results obtained are discussed in the framework of the model where the charge state formation occurs in electron exchange between sputtered atoms and a local surface area excited by the impact of the projectile. (c) 2001 Elsevier Science B.V. All rights reserved.
\end{abstract}

Keywords: Secondary ion mass spectrometry; Ion emission; Ion bombardment; Ion-solid interactions; Sputtering; Niobium; Tantalum; Semi-empirical models and model calculations

\section{Introduction}

It is well known that the bombardment of metals by keV energy atomic ions results in emis-

\footnotetext{
${ }^{*}$ Corresponding author. Tel.: +32-3-820-2363; fax: +32-3820-2376.

E-mail address: belikh@uia.ua.ac.be (S.F. Belykh).
}

sion of secondary particles (including polyatomic ones) in different charge states [1]. Ion fractions in the total flux of sputtered atoms depend on many factors, such as the electronic structure of a surface, species of particles, their velocities and escaping angle [2]. According to the existing theoretical models, the dominant process of ion formation is the interaction between the outgoing particle and the solid surface. The interaction is 
considered either as a time-depending perturbation [3] or as a certain electron exchange process [4-9]. In all these models [3-9] are assumed that the ionisation probability of sputtered atoms does not depend on the parameters of projectiles.

A majority of the secondary particles is formed as a result of several many-body collisions in the subsurface region of the solid. When leaving the surface they interact with a local surface area where both the electronic subsystem and the lattice structure are perturbed by a continuing cascade of collisions. Therefore, a theoretical description of the charge state formation processes is not easy, first of all, because of an uncertainty of electronic properties of a local surface area from which the emission of secondary atomic ions occurs. For the first time the attempts to explain the effect of the electronic subsystem excitation on the ionisation probability of sputtered atoms were undertaken by Šroubek $[10,11]$. However, the question "whether perturbation of the target electronic subsystem affects on the charge state formation of sputtered atoms" is still under discussion.

Excitation of the electronic subsystem under solid bombardment by keV-energy atomic ions plays a principal role in such phenomena as kinetic ion-electron emission [12], multiply charged ion emission [2] and emission of metastable excited neutral atoms [13]. The electronic subsystem is excited by energetic collisions with involvement of the projectile and fast recoil atoms, leading to the formation of vacancies in inner shells of target atoms [2,12]. For the case of transition metals, most of these excitations manifest themselves as the d-band holes [13]. In principle, such a nonequilibrium excitation of the electronic subsystem may affect the processes of the charge state formation of sputtered atoms. But this effect is only to be essential if the sputtered atoms leave the surface before the excitation is able to relax. In this context, it is important to know the relaxation time $t_{0}$ of the electronic excitations.

As a rough estimate for relaxation time of oneelectron excitations in metals, the value $t_{0} \sim 10^{-16}$ $\mathrm{s}$, obtained from simple kinematics considerations, is usually used. Since $t_{0} \ll \tau_{0} \quad\left(\tau_{0} \sim 10^{-12} \mathrm{~s}\right.$ is the time of collision cascade development) it seems that for metals the effect of the electronic sub- system excitation on charge state formation of sputtered atoms is inessential owing to the fast relaxation of the excitation. However, collective effects of electron gas in metals (for instance, dynamical screening of electrons [14]) can weaken the electron-electron interactions, resulting in raising $t_{0}$. This is justified by direct measurements demonstrating that the relaxation time of the electronic subsystem after its excitation in the gold sample by a femtosecond pulse of laser radiation has the value of $t_{0} \sim 5 \times 10^{-13} \mathrm{~s}$ [15]. One could expect that for other metals $t_{0}$ will not be less. As measured by Sun et al. [15], $t_{0} \sim \tau_{0}$ is in agreement with the values calculated within the different models, that take into consideration the dynamical screening of electrons in metals [16,17]. It should be noted that under the ion bombardment, $t_{0}$ will be not less because the electronic excitation is caused by a collision cascade rather than a single pulse. Indeed, in this case a bombarding ion and fast recoil atoms are able to generate the electron-hole pairs in inelastic collisions with electrons and target atoms until these collisions become energetically impossible. The relaxation time of d-band holes produced by the collision cascade was estimated by Wucher and Šroubek [13], according to which $t_{0}$ is $10^{-13} \mathrm{~s}$.

On the basis of all this, it is quite natural to suppose that the electronic subsystem of a metal within the collision cascade will be in the nonequilibrium excited state while energetic collisions involving the projectile and the fast recoil atoms occur.

Until now, there are no direct experimental studies of an effect of non-equilibrium excitation of the metal electronic subsystem on the ionisation probability of sputtered atoms. Nevertheless, such studies are of both fundamental and applied interest; in principle, they can be carried out if characteristics of atomic ions sputtered for different degrees of electronic subsystem excitation are compared. In any experiment, there are several parameters allowing a change of the degree of electronic excitation. For example, one can vary the primary energy of a given atomic projectile or, one can change the species of the atomic projectile at a fixed primary energy. Another possibility is connected with the change of the number of atoms 
in the projectile while keeping constant the projectile velocity.

In our opinion, the latter case is more attractive for such studies. Indeed, in comparison with the atomic bombardment, the molecular one, as a rule, results in a greater yield of sputtered particles per one atom of the projectile, in other words, in nonadditive sputtering. In the 1970 s it was found [18] that under bombardment of heavy metal targets ( $\mathrm{Ag}, \mathrm{Au}, \mathrm{Pt}$ ) by heavy atomic and molecular ions $\left(\mathrm{As}^{+}, \mathrm{As}_{2}^{+}, \mathrm{Sb}^{+}, \mathrm{Sb}_{2}^{+}, \mathrm{Sb}_{3}^{+}, \mathrm{Bi}^{+}, \mathrm{Bi}_{2}^{+}\right)$with $E_{0}=10-250 \mathrm{keV}$ per atom the non-additive total yield $Y_{m}$ of sputtered particles is observed. Here $Y_{m}=\sum_{n} Y_{n, m}$ is the average number of sputtered atoms, molecules and clusters in different charge states per one incident ion. Non-additive sputtering is usually described by the non-additive factor (or the enhancement factor) $K_{m, m^{\prime}}=m^{\prime} Y_{n, m} / m Y_{n, m^{\prime}}$, where $Y_{n, m}$ and $Y_{n, m^{\prime}}$ are the yields of $n$-atomic particles, which are measured at the same projectile velocity and $m$ and $m^{\prime}$ are the numbers of atoms in the projectiles. Using the definition of $K_{m, m^{\prime}}$, the results of Ref. [18] mean that under the metal bombardment by $m$-atomic projectiles $Y_{m}$ is more than by a factor of $m$ higher as compared to the yield $Y_{1}$ under the bombardment by the atomic ions $\left(m^{\prime}=1\right)$. According to the results of Refs. [18-21], for $E_{0}>10 \mathrm{keV}$ per atom the values of $K_{2,1}=Y_{2} / 2 Y_{1}$ do not exceed several units. For example, in Ref. [18] it is shown that under the bombardment of $\mathrm{Au}$ (an element characterised by a greater yield of sputtering [22]) with $\mathrm{Bi}^{+}$and $\mathrm{Bi}_{2}^{+}$ ions $K_{2,1}$ is equal to 3.9 at $45 \mathrm{keV}$ per atom and 2.5 at $30 \mathrm{keV}$ per atom. There are no available measurements of $K_{2,1}$ for $E_{0}<10 \mathrm{keV}$ per atom. However, the extrapolation of these data to the range of lower $E_{0}$ values predicts even lower values of $K_{2,1}$.

Taking into account the features of molecular bombardment, different assumptions have been made to explain reasons for non-additive sputtering. The unique character of molecular bombardment resides with the fact that it ensures spatial localisation and time synchronisation of projectile atom collisions with the surface. The following factors might be responsible for increasing the sputtering yield in conditions of molecular ion bombardment.
- Practically simultaneous impact of the constituent atoms onto a small area of the surface $\left(\sim 10^{-16} \mathrm{~cm}^{2}\right)$ increases the density of energy released in the subsurface region of the solid (hence, causing non-linear cascades [23]).

- Each subsequent atom of the molecular ion interacts over the time $\Delta t \leqslant 10^{-15} \mathrm{~s}$ with a local surface area excited by the impact with a frontrunner atom. This means that the subsequent atom can be considered as a "probe" sampling the surface before the excitations of both the electronic subsystem and the lattice structure to relax. Such excitations can decrease the binding energy of atoms in the cascade region increasing the sputtered atom yield [18]. On the other hand, they can change the conditions of charge state formation leading to the raise of the atomic ion yield.

It has been found that neutral atoms dominate in the total yield $Y_{m}$ of secondary particles [24-28]. Hence, determined in Refs. [18-21], non-additive yields $Y_{m}$ are mainly defined by non-additive sputtering of neutral atoms. Atomic ions constitute a large fraction in the total flux of secondary charged particles. In the case of bombardment of $\mathrm{Nb}$ and $\mathrm{Ta}$ targets $(\mathrm{Nb}$ and $\mathrm{Ta}$ have lower yields of sputtering [22] than gold) by ions of $\mathrm{Au}_{m}^{-}$ $(1 \leqslant m \leqslant 3)$ with $E_{0}=6 \mathrm{keV}$ per atom the following data are obtained: $K_{3,1}\left(\mathrm{Nb}^{+}\right)=7 ; K_{2,1}\left(\mathrm{Nb}^{+}\right)=$ $3 ; K_{3,1}\left(\mathrm{Ta}^{+}\right)=3.5 ; K_{2,1}\left(\mathrm{Ta}^{+}\right)=2.5$, respectively [29-32]. It is important that these values of $K_{m, m^{\prime}}$ obtained for atomic ions are comparable or even exceed the ones determined for the gold sputtering yield [18-21] at higher values of $E_{0}$. Such an excess of $K_{m, m^{\prime}}$ for atomic ions compared to those of neutral particles leads to the assumption that molecular bombardment not only leads to the increase of the sputtered atom yield but also changes the conditions of charge state formation resulting in an increase of the ionisation probability of sputtered atoms.

These reasons for the non-additive yields of atoms and atomic ions under molecular bombardment of metals need to be justified experimentally. Until now, there are no final replies to the following questions. 
- Does the molecular bombardment effect the charge state formation process of sputtered atoms?

- What are the contributions of the processes of sputtering and charge state formation to nonadditive yield of atomic ions?

In the present work a method to study the effect of electronic subsystem excitation in solids on the ionisation probability of sputtered atoms is proposed. It was used for the analysis of the kinetic energy distributions of $\mathrm{Nb}^{+}$and $\mathrm{Ta}^{+}$ions sputtered from $\mathrm{Nb}$ and $\mathrm{Ta}$ targets by atomic and molecular $\mathrm{Au}_{m}^{-}$ions $(1 \leqslant m \leqslant 3)$ with an energy of $E_{0}=6 \mathrm{keV}$ per atom. The results obtained highlight the important role of the metal electronic excitations in ion emission processes.

\section{Experimental conditions, method and results}

Important information on the features of the non-additive sputtering of solids can be obtained from the comparison of the kinetic energy distributions $f_{n, m}(E)$ of secondary ions. In our previous works [29-32] the energy distributions $f_{n, m}(E)$ of $\mathrm{Nb}_{n}^{+}(1 \leqslant n \leqslant 16)$ and $\mathrm{Ta}_{n}^{+}(1 \leqslant n \leqslant 13)$ ions sputtered from $\mathrm{Nb}$ and $\mathrm{Ta}$ targets by $\mathrm{Au}_{m}^{-}(1 \leqslant m \leqslant 3)$ projectiles with the energy $E_{0}=6 \mathrm{keV}$ per atom were measured under identical experimental conditions.

The experimental SIMS instrument used for the measurements has been described in detail elsewhere $[30,32]$. Here we only discuss the experimental conditions and the cleaning procedures used for the preparation of the target surfaces. It is a well-known fact that the reaction of metals with electronegative elements (for example, oxygen) usually proceeds through chemisorption to the formation of surface oxides. This changes the electronic properties of metallic surfaces and can result in dramatic enhancement of positive secondary ion yields relative to those from clean metals [2]. That is why for a reliable comparison of secondary ion yields determined under the bombardment by the atomic and molecular projectiles, the clean conditions on the target surfaces are required. Niobium and tantalum were chosen as targets owing to their high melting temperatures. The cleaning procedures included both the heating and the ion sputtering of the targets. These were cleaned by heating to high temperatures $(2400 \mathrm{~K}$ for $\mathrm{Nb}$ and $2600 \mathrm{~K}$ for $\mathrm{Ta}$ ) and by the subsequent $\mathrm{Au}^{-}$ion bombardment for several hours. The yields of $\mathrm{NbO}^{+}$and $\mathrm{TaO}^{+}$molecules sputtered from $\mathrm{Nb}$ and $\mathrm{Ta}$ surfaces by the $\mathrm{Au}^{-}, \mathrm{Au}_{2}^{-}$and $\mathrm{Au}_{3}^{-}$projectiles were controlled before and after the cleaning procedures. They were observed to drop by more than three orders of magnitude after the cleaning procedures applied. Since the probability of chemical reactions increases on hot solid surfaces, we believe that this drop in the $\mathrm{NbO}^{+}$ and $\mathrm{TaO}^{+}$yield indicates a corresponding decrease in the oxygen concentration on the target surfaces. The target surfaces prepared in such a way were believed to be "clean surfaces". During the measurements the temperatures of the $\mathrm{Nb}$ and $\mathrm{Ta}$ samples were maintained at 2100 and $2300 \mathrm{~K}$, respectively, and the residual gas pressure did not exceed $1 \times 10^{-5} \mathrm{~Pa}$.

In such experimental conditions it was found that the shape of the distributions $f_{n, m}(E)$ is defined by the species of secondary ions used. For a given secondary ion the shape of curves $f_{n, m}(E)$ depends on the number $m$. It essentially differs for the atomic bombardment and the molecular one. In this work we analysed only the energy distributions $f_{1, m}(E)$ of atomic $\mathrm{Nb}^{+}$and $\mathrm{Ta}^{+}(n=1)$ ions. The analysis of the energy distributions $f_{n, m}(E)$ of $n$-atomic ions will be given in an other article.

The main point of the method proposed here is the study of an effect of non-equilibrium excitation of an electronic subsystem of metals on the ionisation probability of sputtered atoms. This is realised by a comparison of the energy distributions of atomic ions sputtered from clean metal surfaces by atomic and molecular projectiles.

In general, the kinetic energy distribution $f_{1,1}(E, \theta)$ of sputtered atomic ions is defined according to [1]:

$f_{1,1}(E, \theta)=F_{1,1}(E, \theta) P^{+}(E, \theta)$,

where $F_{1,1}(E, \theta)$ is the energy distribution of sputtered atoms and $P^{+}(E, \theta)$ is the probability of their ionisation. For atoms, the expression $F_{1,1}(E, \theta)$ 
follows from the Sigmund cascade theory of sputtering [33] is usually used as:

$F_{1,1}(E, \theta)=\frac{E}{\left(E+U_{0}\right)^{3}} \cos \theta$,

where $U_{0}$ is the binding energy. The function $F_{1,1}(E, \theta)$ depends on the binding energy $U_{0}$, the kinetic energy $E$, and the escape angle $\theta$ of sputtered atoms. It does not depend on the species and the energy $E_{0}$ of the projectile. According to conventional theoretical ideas [3-9], the ionisation probability of sputtered atoms is defined by tunnelling of an electron between the metal surface and the emitted particle. In the framework of the electron tunnelling theory [7], the ionisation probability of sputtered atoms is described by the exponential function:

$P^{+}(E, \theta) \propto \exp \left(-v_{0} / v_{z}\right)$,

where $v_{0}$ is the parameter characterising the ionisation efficiency, $v_{z}=v \cos \theta$, where $v=\sqrt{2 E / M}$ and $M$ are the velocity and mass of the sputtered atom, respectively. The parameter $v_{0}$ depends on the ionisation potential $I$ of the sputtered atoms and on the work function $\varphi$ of the metal surface. In the interpretation we tentatively assume the validity of Eq. (3) also in our case.

As compared with atomic ion bombardment of metals, the molecular one results in the enrichment of the energy distribution by low energy atoms while the higher energy part of this distribution does not practically change [19]. In this connection, we assume that the distribution $F_{1, m}(E, \theta)$ for the molecular bombardment does not change over the energy range $E>5 \mathrm{eV}$ as compared with that for the atomic bombardment (i.e. $F_{1,1}(E, \theta)=$ $\left.F_{1,2}(E, \theta)=F_{1,3}(E, \theta)\right)$. Thus Eq. (1), after the substitution of experimental distributions $f_{1, m}(E, \theta)$ and the calculated ones $F_{1, m}(E, \theta)$, can provide information on the value of $v_{0}$.

The energy distributions of $\mathrm{Nb}^{+}$and $\mathrm{Ta}^{+}$ions (Fig. 1a, b) sputtered from clean targets by $\mathrm{Au}_{m}^{-}$ $(m=1-3)$ projectiles with the energy $E_{0}=6 \mathrm{keV}$ per atom [29-32] were used as the distributions $f_{1, m}(E, \theta)$. For experiments in Refs. [29-32] $\theta=0^{\circ}$. To calculate the distribution $F_{1, m}(E, \theta)$ according to Eq. (2) the value of $U_{0}=3 \mathrm{eV}$ was used.
For $\mathrm{Nb}^{+}$and $\mathrm{Ta}^{+}$ions, the dependence of $\ln P^{+}=\ln \left\{f_{1, m}(E, \theta) / F_{1, m}(E, \theta)\right\}$ on $v_{z}^{-1}$ are plotted in Fig. 2a and b. One can see that the dependence is approximated satisfactorily by straight lines. This provides evidence that the charge state formation of $\mathrm{Nb}^{+}$and $\mathrm{Ta}^{+}$ions, both under the atomic bombardment and the molecular one, is described by Eq. (3). The slopes of the straight lines, according to Eq. (3), are equal to the corresponding values of $v_{0}$ and characterise the efficiency of the electron tunnelling process. In the case of $\mathrm{Nb}^{+}$ions the values of $v_{0} \times 10^{-4}\left(\mathrm{~s} \mathrm{~m}^{-1}\right)$ are equal to $0.3796 \pm 0.0137,0.3324 \pm 0.0176$ and $0.2918 \pm 0.0123$ for $\mathrm{Au}^{-}, \mathrm{Au}_{2}^{-}$and $\mathrm{Au}_{3}^{-}$, respectively. For $\mathrm{Ta}^{+}$ions the values of $v_{0} \times 10^{-4}\left(\mathrm{~s} \mathrm{~m}^{-1}\right)$ are equal to $0.5528 \pm 0.0064\left(\mathrm{Au}^{-}\right), 0.4006 \pm$ $0.0054\left(\mathrm{Au}_{2}^{-}\right)$and $0.2956 \pm 0.0071\left(\mathrm{Au}_{3}^{-}\right)$. Thus, these data demonstrate that under molecular ion bombardment the ionisation efficiency of sputtered atoms increases in comparison with the atomic one. This effect results in an increasing yield of secondary atomic ions, especially low energy ones.

This conclusion can be also obtained in another way. The energy distributions $f_{1, m}(E)$ of $\mathrm{Nb}^{+}$and $\mathrm{Ta}^{+}$ions (Fig. 1a, b) make it possible to establish a relation between non-additive sputtering and energy $E$ of sputtered particles. The quantitative characteristic of this relation can be defined as the differential non-additive factor $k_{m, m^{\prime}}=m^{\prime} f_{n, m}(E) /$ $m f_{n, m^{\prime}}(E)$. Thus, $k_{m, m^{\prime}}$ is equal to the ratio of yields of sputtered atomic ions with the given energy $E$ per atom of the projectile. The factors $K_{m, m^{\prime}}$ and $k_{m, m^{\prime}}$ are connected with each other by the simple relation: $K_{m, m^{\prime}}=1 /\left(E_{\max }-E_{\min }\right) \int_{E_{\min }}^{E_{\max }} k_{m, m^{\prime}}(E) \mathrm{d} E$ or using the mean-value theorem: $K_{m, m^{\prime}}=k_{m, m^{\prime}}\left(E^{*}\right)$ where $E_{\min }<E^{*}<E_{\max }$.

The functions $k_{3,1}(E)$ and $k_{2,1}(E)$ for $\mathrm{Nb}^{+}$and $\mathrm{Ta}^{+}$ions within the energy range of $E(1<E<25$ $\mathrm{eV}$ ) are presented in Fig. 3a and b. As is seen from Fig. 3a, for $\mathrm{Nb}^{+}$ions the curve $k_{3,1}(E)$ has a maximum value of $k_{\max }=10.5$ at $E=1 \mathrm{eV}$ and then decreases monotonously with $E$, reaching the value $k_{\min }=6$ at $E=25 \mathrm{eV}$. In this case the following inequality is valid $k_{\max }>K_{3,1}>k_{\min }$, where the non-additive factor $K_{3,1}=7$ is measured for the $\mathrm{Nb}^{+}$ion flux over the energy range $0<E<$ $300 \mathrm{eV}$ [32]. 

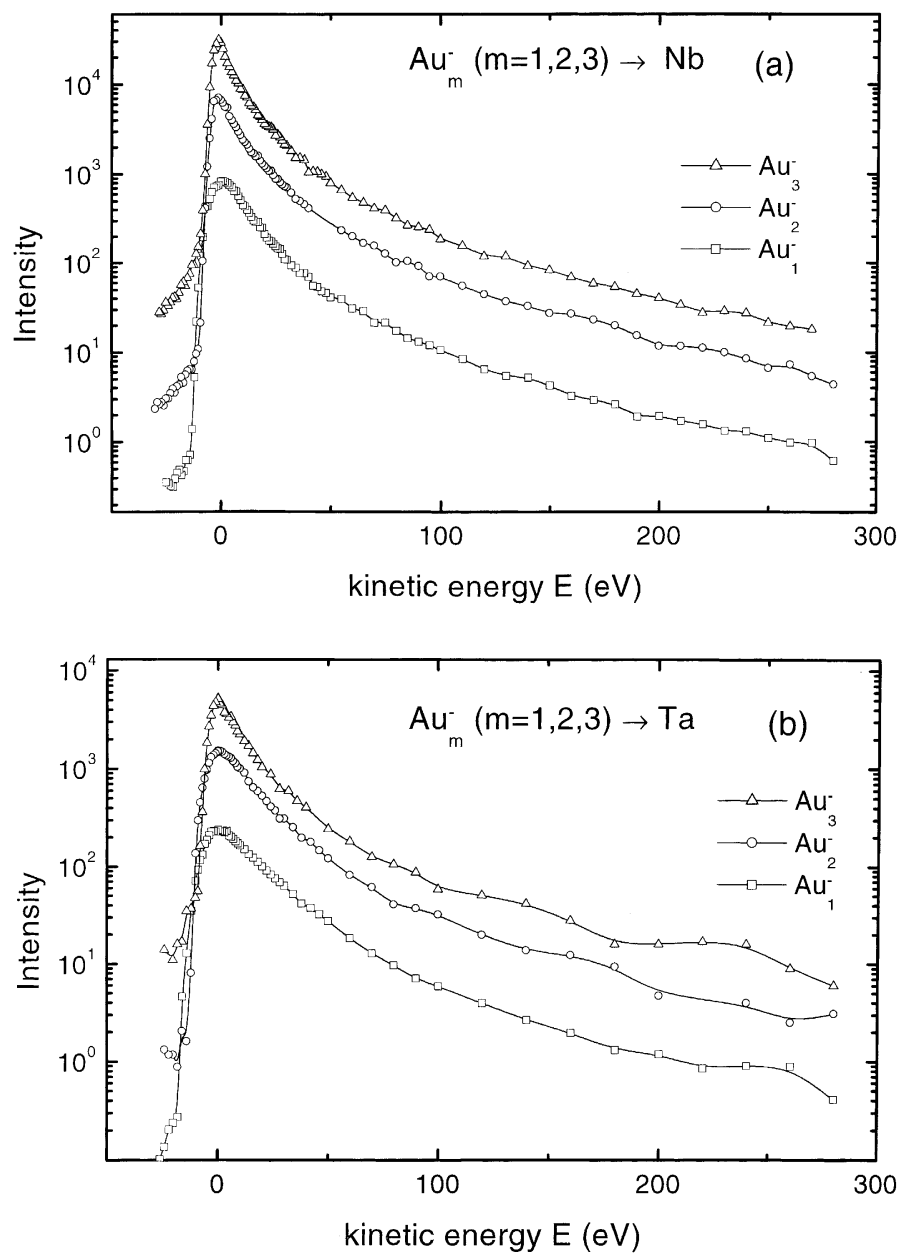

Fig. 1. Kinetic energy distributions of atomic (a) $\mathrm{Nb}^{+}$and (b) $\mathrm{Ta}^{+}$ions sputtered from niobium and tantalum targets by atomic and molecular $\mathrm{Au}_{m}^{-}$projectiles $(1 \leqslant m \leqslant 3)$ with the primary energy of $6 \mathrm{keV}$ per atom. For the convenience of a curve shape comparison all energy distributions are displaced arbitrary to each other along the ordinate axis. For all distributions the levels of background are the same and ones are equal 1-3 count/s.

The dependence $k_{m, m^{\prime}}(E)$ is also sensitive to the number of atoms in the projectile. The change of $m$ from $m=2$ to $m=3$ (at $m^{\prime}=1$ ) results in increasing $k_{m, m^{\prime}}\left(k_{3,1}>k_{2,1}\right)$ and the difference between $k_{\max }$ and $k_{\min }$. Indeed, for $\mathrm{Nb}^{+}$ions the values of $k_{\max }\left(k_{\max }=8\right.$ and $\left.k_{\max }=10.5\right)$ exceed those of $K_{2,1}=3$ and $K_{3,1}=7$, respectively. For $\mathrm{Ta}^{+}$ions the values of $k_{\max }\left(k_{\max }=3.3\right.$ and $\left.k_{\max }=6.7\right)$ also exceed those of $K_{2,1}=2.5$ and $K_{3,1}=3.5$. Thus, as compared with the atomic bombardment, the molecular one results in increasing the yield of slow $\mathrm{Nb}^{+}$and $\mathrm{Ta}^{+}$ions. The higher the number $m$ is the higher the yield becomes.

As established by numerous studies, under bombardment of metals by $\mathrm{keV}$ atomic projectiles [2], the yield of sputtered atomic ions is a factor of $10^{3}-10^{5}$ less than that of neutral atoms. The difference in the yields of neutral atoms and atomic ions is connected with the process of charged state formation. As mentioned above, the ionisation probability of sputtered atoms is defined by the tunnelling of an electron between the metal surface and the emitted particle. In accordance with Ref. 

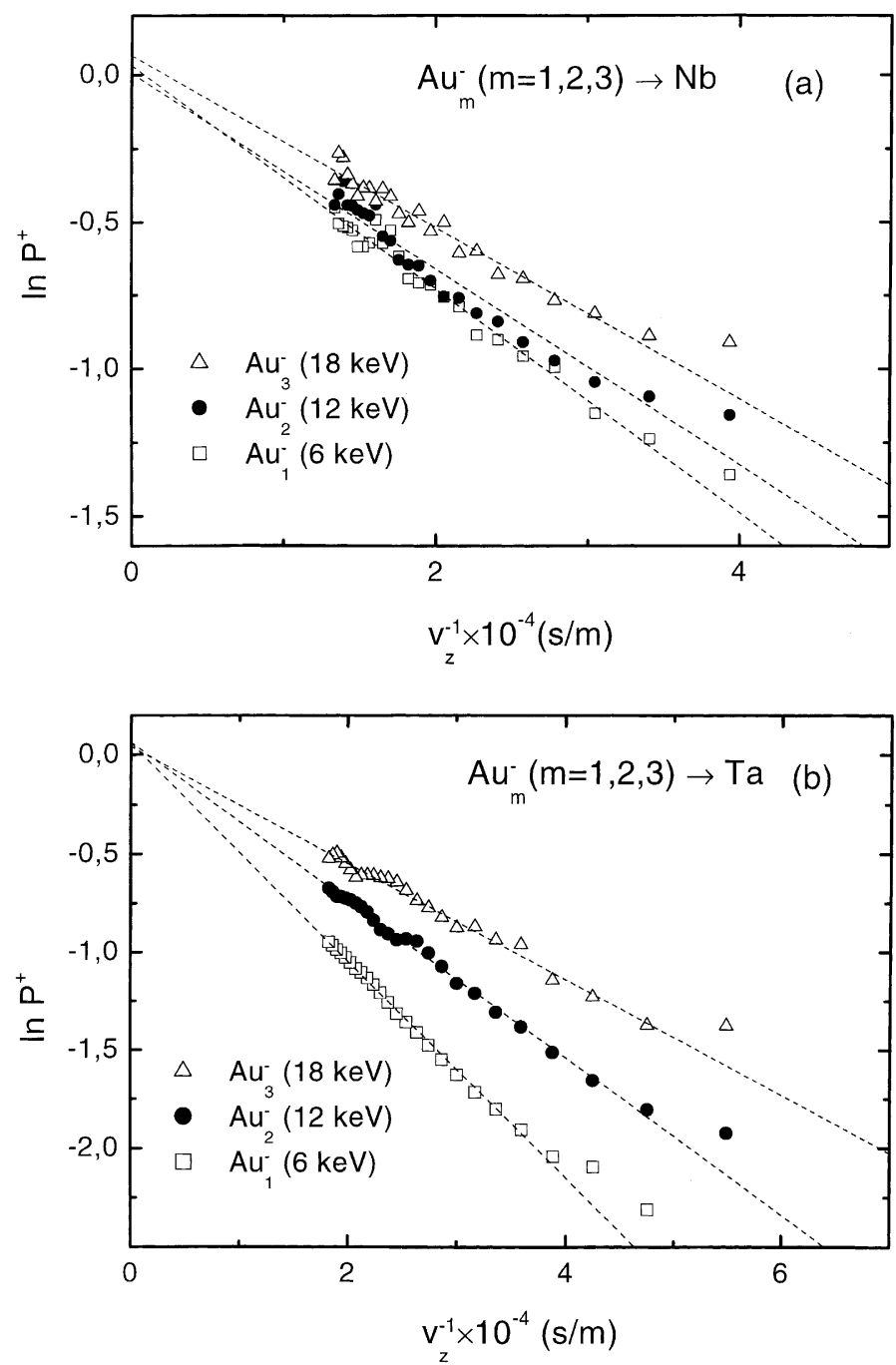

Fig. 2. Ionisation probability logarithm vs $v_{z}^{-1}$ for atomic (a) $\mathrm{Nb}^{+}$and (b) $\mathrm{Ta}^{+}$ions sputtered from niobium and tantalum targets by atomic and molecular $\mathrm{Au}_{m}^{-}$projectiles $(1 \leqslant m \leqslant 3)$ with the energy of $6 \mathrm{keV}$ per atom.

[2], the ionisation probability essentially decreases with the decrease of energy $E$. However, in our case the functions $k_{2,1}(E)$ and $k_{3,1}(E)$ for $\mathrm{Nb}^{+}$and $\mathrm{Ta}^{+}$ions demonstrate an opposite tendency: the ratio of yields of atomic ions increases, when $E$ decreases. Moreover, this tendency becomes more pronounced with the rise of $m$.

Thus, the molecular ion bombardment creates on the metal surface conditions favouring the increase of the ionisation probability of sputtered atoms, especially of atoms with low $E$.

\section{Discussion}

Based on the results of the analysis, the physical reasons for the increase in the ionisation probability of atomic particles can be defined. Indeed, according to Eq. (3), $P^{+}(E, \theta)$ depends on the characteristics of sputtered atoms (the ionisation potential $I$, the velocity $v$ and the escape angle $\theta$ ) as well as on the electronic properties of the surface (the work function $\varphi$ ). For the given species of secondary atomic ions each of the dependence 

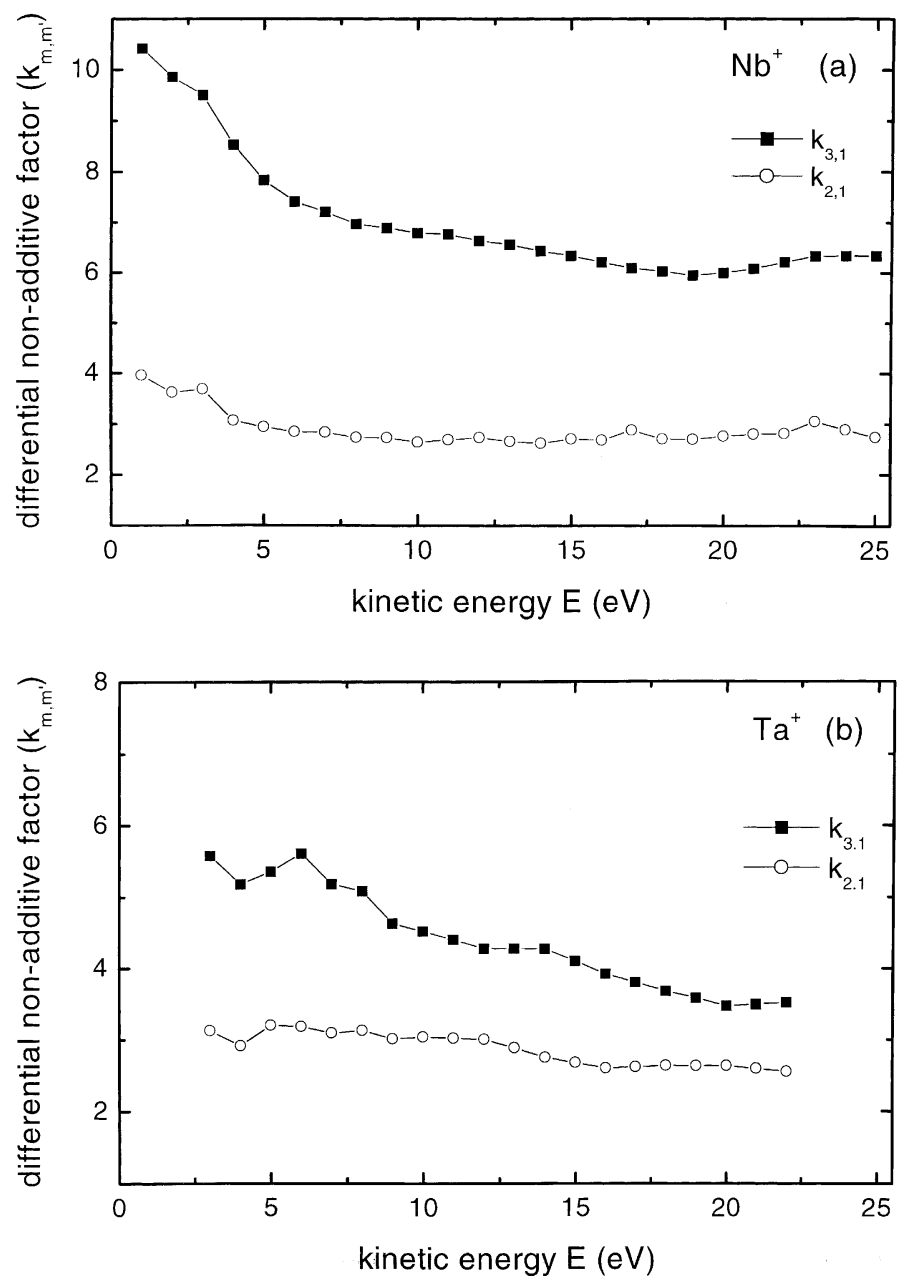

Fig. 3. Differential non-additive factors $k_{3,1}$ and $k_{2,1}$ vs the kinetic energy $E$ of sputtered (a) $\mathrm{Nb}^{+}$and (b) $\mathrm{Ta}^{+}$ions.

$\ln P^{+}$on $v_{z}^{-1}$ (Fig. 2a, b) corresponds to the same values of $I, v$ and $\theta$. Therefore, the reasons for the increase in the ionisation efficiency for the molecular bombardment, as compared to the atomic one, are to be connected with the change of the electronic properties of a local surface area from which the emission of atomic ions occurs. In our opinion, the change of electronic properties of a local surface area should be considered rather than the change of the work function because $\varphi$ is an integral characteristic of the macroscopic surface and this does not characterise the local nature of the processes of charge state formation. The change of electronic properties of the local surface area is caused by the electronic subsystem excitation resulting from the ion bombardment of the metal.

Non-equilibrium electronic excitation within the collision cascade can be a result of two different mechanisms. In one of them the projectile ion as well as all fast moving target recoil atoms interact with the conduction band electrons, transferring part of their kinetic energy into the electronic subsystem of the solid. As a result electron-hole pairs form in the conduction band [13,34]. The other mechanism is inelastic collisions between moving target atoms leading to the formation of vacancies in inner shell(s) of the target atoms. The 
decay of such vacancies results in electron emission or/and generation of electron-hole pairs in the conduction band [2]. The appearance of nonoccupied levels below the Fermi level opens an additional channel for the electron exchange. This results in increasing the ionisation probability owing to tunnelling electrons from the sputtered atoms into these levels.

Following Sroubek [10,11], it is convenient to characterise the electronic subsystem excitation by some "effective electron temperature $T_{\text {eff }}$ ". It is necessary to stress that $T_{\text {eff }}$ is not the real thermodynamic temperature of the metal target. A parameter $T_{\text {eff }}$ characterises the shift of the electron energy distribution in the conduction band of the metal from its equilibrium state. The value of $T_{\text {eff }}$ depends sensitively on the substrate parameter $\gamma$, which characterises the change of the width $\Delta$ of the atomic level with the distance from the surface $z$ [2]: $\Delta=\Delta_{0} \exp (-2 \gamma z)$ where $\Delta_{0}$ is the width of the atomic level on the surface $(z=0)$. According to Refs. $[10,11]$ the final charged state of atoms sputtered from the clean metal surface, in the high temperature limit, becomes:

$P^{+}=\exp \left[-\frac{\varepsilon_{\mathrm{F}}-\varepsilon_{\mathrm{a}}\left(z_{0}\right)}{k_{\mathrm{B}} T_{\mathrm{eff}}}\right]$,

where $\varepsilon_{\mathrm{F}}$ is the Fermi energy, $\varepsilon_{\mathrm{a}}\left(z_{0}\right)$ is the energy of the atomic level at the freezing point $z_{0}$ [8] and $k_{\mathrm{B}}$ is the Boltzmann constant, the energies being counted from the vacuum level. We remind, that for $z<z_{0}$, closer to the surface, the charge state is nearly the equilibrium one. Outside this region the electron exchange is considered being frozen out. Let us further define $z_{0}$ as the distance that the sputtered atom with the normal velocity $v_{z}$ reaches within the relaxation time. Then $z_{0}=v_{z} t_{0}$. In the case of emission from clean metals, the dependence of the atomic energy level $\varepsilon_{\mathrm{a}}$ on $z$ can be well approximated for larger $z$ by the image charge energy [10]:

$\varepsilon_{\mathrm{a}}(z)=\varepsilon_{\mathrm{a}}(\infty)+e^{2} / 4 z$,

where $\varepsilon_{\mathrm{a}}(\infty)=-I$ is the energy of the atomic level at infinity and $e$ is the electron charge. Then, inserting Eq. (5) in Eq. (4), for the dependence of $P^{+}$ on $v_{z}$ we obtain the equation:
$P^{+} \propto \exp \left[-\frac{e^{2}}{4 v_{z} t_{0} k_{\mathrm{B}} T_{\mathrm{eff}}}\right]$

which coincides with Eq. (3) if $v_{0}=e^{2} / 4 t_{0} k_{\mathrm{B}} T_{\text {eff }}$.

Using Eq. (6) the values of $T_{\text {eff }}$ can be derived from the experimental data (Fig. 2a, b). Putting $t_{0} \approx 5 \times 10^{-13} \mathrm{~s}$ as in Ref. [15], one obtains 2200, 2500 and $2850 \mathrm{~K}$ for $\mathrm{Nb}$ sputtering at $m=1,2$, and 3 , respectively. For the $\mathrm{Ta}$ sputtering the values of $T_{\text {eff }}$ become 1500,2050 and $2800 \mathrm{~K}$ at $m=1,2,3$, respectively.

For the $\mathrm{Nb}$ and $\mathrm{Ta}$ sputtering the functions $T_{\text {eff }}(m)$ are presented in Fig. 4. These data show the following features:

- For the atomic bombarding $(m=1)$ the ratio $T_{\text {eff }}(\mathrm{Nb}) / T_{\text {eff }}(\mathrm{Ta})$ approximates 1.5 .

- $T_{\text {eff }}$ increases approximately linearly with the rise of $m$.

- The slopes of $T_{\text {eff }}(m)$ are different for the $\mathrm{Nb}$ and Ta sputtering (0.46 and 0.68 , respectively), being less than unit.

The degree of the electronic subsystem excitation, and, as a consequence, $T_{\text {eff }}$ is connected with inelastic energy losses of projectiles and fast recoil atoms moving inside the cascade region. The inelastic energy losses are proportional to the velocity $w$ of the moving particle, and $T_{\text {eff }}$ can be estimated from the formula obtained by Lörinčík et al. [34]:

$k_{\mathrm{B}} T_{\mathrm{eff}}=\frac{2 \gamma \hbar w}{\pi}$,

where $\hbar$ is Plank's constant. Taking into account that for the atomic projectile bombardment $(m=1) T_{\text {eff }}(\mathrm{Nb})>T_{\text {eff }}(\mathrm{Ta})$, we must assume that the main contribution to the electronic excitation result from fast target recoils. The average energy transferred by a gold atom to the recoil is $\bar{E}=2 \mu /(\mu+1)^{2} E_{0}$, where $\mu$ is the mass ratio. The average velocities $w$ of recoils in the $\mathrm{Nb}$ and $\mathrm{Ta}$ substrates are $7.3 \times 10^{4}$ and $5.6 \times 10^{4} \mathrm{~m} \mathrm{~s}^{-1}$, respectively. Using these values and $\gamma=10^{10} \mathrm{~m}^{-1}$ [34] for the both substrates (assuming the electronic properties of $\mathrm{Nb}$ and $\mathrm{Ta}$ to be identical) we find estimates for $T_{\text {eff }}$ to be equal $2800 \mathrm{~K}$ for Ta 


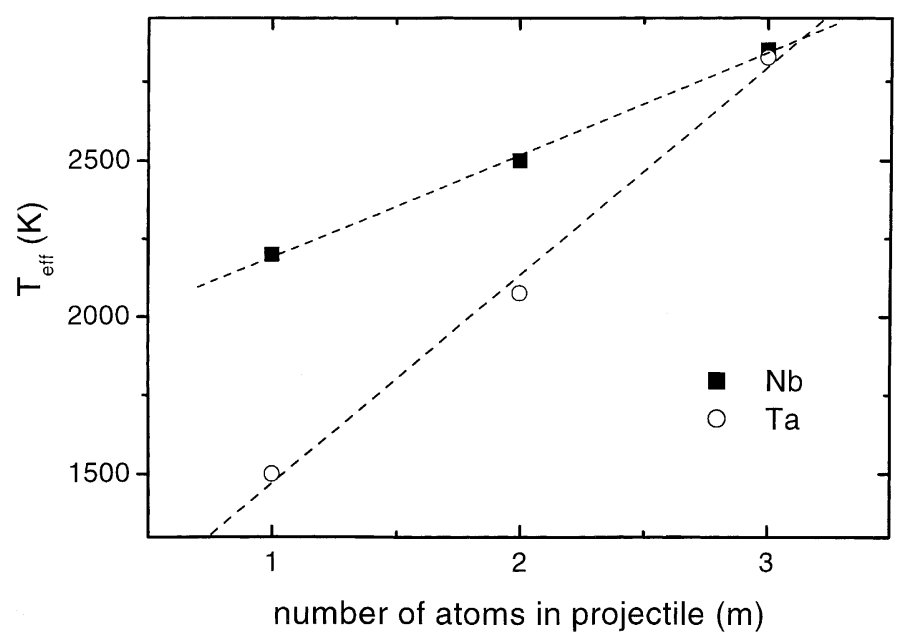

Fig. 4. Dependence of the effective temperature $T_{\text {eff }}$ on the number atoms in $\mathrm{Au}_{m}^{-}$projectiles for $\mathrm{Nb}$ and $(\bigcirc)$ Ta sputtering.

sputtering, and $3600 \mathrm{~K}$ for the $\mathrm{Nb}$. The temperature ratio for $T_{\text {eff }}(\mathrm{Nb}) / T_{\text {eff }}(\mathrm{Ta})=1.3$, i.e. is close to the experimental value 1.47 .

The rise of $T_{\text {eff }}$ with increasing $m$ can be easily understood, because the more atoms constitute the projectile the more fast recoils, exciting the electronic subsystem, they produce. This effect is "inverse" non-additive because the slopes of the $T_{\text {eff }}(m)$ functions are less than unity. This means that the electronic excitation produced by the impact of the molecular projectile is less the sum of the excitations induced by the independent constituents. It is clear that $T_{\text {eff }}(m)$ must depend in a complicated way on trajectories of $m$ constituents of the projectile. However, a simple qualitative explanation of the inverse non-additive effect is possible in terms of a "clearing the way" effect [3539]. Indeed, a frontrunner atom of the molecular projectile kicks the target atoms away from the trajectories of the subsequent projectile(s). As a result, the backrunner atoms collide with the target ones at larger collision parameters. Therefore, an amount of the energy transferred by the backrunner(s) to recoil atoms becomes less, and as a consequence, their velocities will be less than the velocities of recoils produced by the frontrunner. The longer time the molecular constituents move together in the target matter the more pronounced should be the inverse non-additive effect in the electronic subsystem excitation. The last must be realised for the bombardment of light targets by heavy projectiles. On the contrary, the less the mass of a projectile constituents is with respect to the mass of the target atoms, the faster the molecule disintegrates on its path, the less pronounced the "clearing the way" effect should be.

Eq. (6) also allows one to describe the behaviour of the differential non-additive factor $k_{m, m^{\prime}}$ with variation of energy $E$ of the secondary ions and the change of the number $m$ of atoms in the projectile. Indeed, according to the definition of $k_{m, m^{\prime}}$ using Eqs. (1) and (6) one can write:

$k_{m, m^{\prime}}=\exp \left[\frac{e^{2}}{4 t_{0} k_{\mathrm{B}} T_{\mathrm{eff}}} \frac{\left(b_{m, m^{\prime}}-1\right)}{b_{m, m^{\prime}}} \frac{1}{v_{z}}\right]$,

where $b_{m, m^{\prime}}=T_{\text {eff }}(m) / T_{\text {eff }}\left(m^{\prime}\right)$, and $b_{m, m^{\prime}} \geqslant 1$. Thus, within the approach considered here, $k_{m, m^{\prime}}$ needs to increase with the raise of both $b_{m, m^{\prime}}$ and $v_{z}^{-1}$. The dependence of $\ln k_{m, m^{\prime}}$ on $b_{m, m^{\prime}}$ and $v_{z}^{-1}$ obtained from the data in Fig. 3 are plotted in Fig. 5. It is seen that the experimental dependences are satisfactorily described by linear functions. These results distinctly show that in going from the atomic bombardment to the molecular one an effective increase of the ionisation probability is observed. In the case of the molecular bombardment the increase of the ionisation probability manifests itself for both low energy sputtered atoms $(E<5 \mathrm{eV})$ 

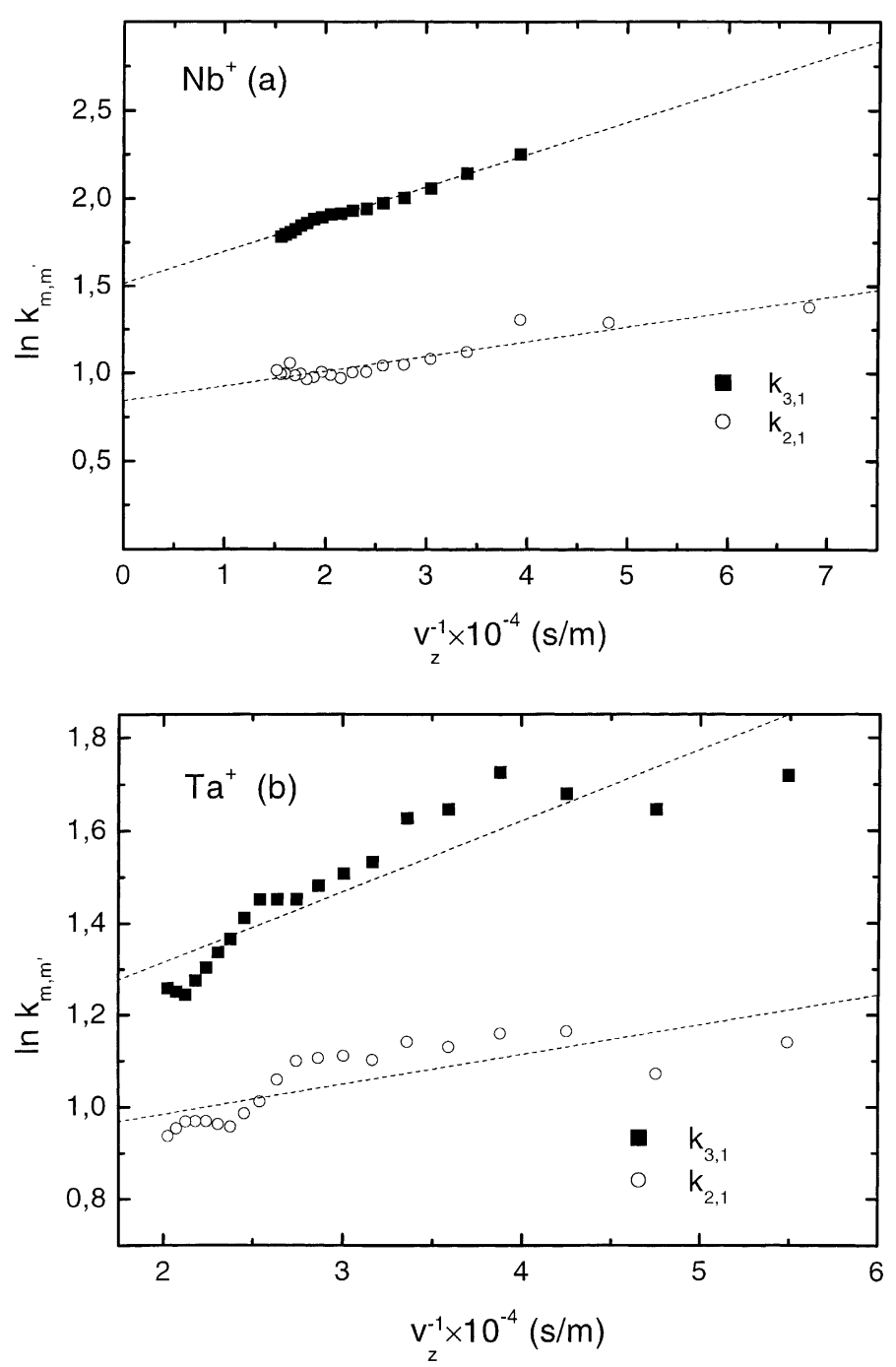

Fig. 5. Differential non-additive factors $k_{3,1}$ and $k_{2,1}$ vs $v_{z}^{-1}$ for the sputtered (a) $\mathrm{Nb}^{+}$and (b) $\mathrm{Ta}^{+}$ions.

and high energy ones, being more pronounced for the lower energy interval. We should admit that the interpretation of these data in terms of the effective electronic temperature is not the only way to treat the increase of the yield of low energy ions sputtered under the molecular bombardment. The determination of a contribution of this effect demands a special study by either direct measurements of the kinetic energy distribution of sputtered neutral atoms or a proper molecular dynamics simulation. Those are out of the scope of the present work.
Taking into account the results obtained one can conclude that the bombardment of metals $(\mathrm{Nb}$ and $\mathrm{Ta}$ ) by the gold atomic and molecular projectiles produces the electronic subsystem excitation which can be described by the effective temperature. Under the molecular ion bombardment the excitation process is inverse non-additive. On the contrary, the charge state formation of sputtered atoms (which demonstrates the exponential dependence of the ionisation probability on the effective temperature) displays itself as the nonadditive process. Thus, non-additive sputtering of 
atomic ions is defined by the joint action of such two factors as the non-additive sputtering of atoms and the non-additive process of their charge state formation. In this case, the non-additive factor $K_{m, m^{\prime}}$ can be represented as a product: $K_{m, m^{\prime}}=$ $K_{\text {sput }} K_{\text {csf }}$ where $K_{\text {sput }}$ is the non-additive sputtering factor and $K_{\text {csf }}$ is the non-additive charge state formation factor.

\section{Concluding remarks}

The comparative study of the kinetic energy distributions of $\mathrm{Nb}^{+}$and $\mathrm{Ta}^{+}$ions sputtered from the respective clean $\mathrm{Nb}$ and $\mathrm{Ta}$ surfaces by $\mathrm{Au}_{m}^{-}$ $(1 \leqslant m \leqslant 3)$ projectiles with the initial energy of $E_{0}=6 \mathrm{keV}$ per atom have been carried out. The main result obtained is experimental demonstration of the effect of the electronic subsystem excitation in the metals on the ionisation probability of sputtered atoms. It is found that, as compared with the atomic ion bombardment, the molecular one leads to the increase of the ionisation probability. It has been shown that this increase is connected with the change of the degree of the electronic subsystem excitation. Such excitation produced by both atomic and molecular ion bombardment results from inelastic collisions of the projectile and the fast recoil atoms in the substrate. As a result of these collisions the electron-hole pairs appear in the conduction band in the subsurface metal region from which the secondary ion emission occurs. The increase of a number of non-occupied levels below the Fermi level in going from the atomic ion bombardment to the molecular one leads to the increase in the ionisation probability owing to tunnelling electrons from the sputtered atoms into these levels.

Thus, one can conclude that the electronic excitation defining by the bombardment conditions affects the ionisation probability of sputtered atoms.

In our opinion, the future experimental and theoretical studies of the effect of the electronic subsystem excitation on the ionisation probability of sputtered atoms will allow to get a better knowledge of the features of secondary ion emis- sion. The results obtained are an applied interest also. Indeed, they indicate the way of increase of the SIMS analysis sensitivity because the use of molecular ions as projectiles and the selection of the optimum sputtering condition result to the increase of the ion yield.

\section{Acknowledgements}

The authors are very grateful to NATO for the support of this work through a Science for Peace grant (project SfP 97.1929). Moreover, S.F.B., V.V.P. and A.A. thank the financial support from IVAP H/10, University of Antwerp (BOF) and FWO (Belgium), respectively. I.A.W. acknowledges the financial support from the SSTC (Belgium). S.F.B., V.V.P. and I.A.W. were formerly members of Arifov Institute of Electronics, Tashkent, Uzbekistan.

\section{References}

[1] W.O. Hofer, in: R. Behrisch, K.Wittmaack (Eds.), Sputtering by Particle Bombardment III, Springer, Berlin, 1991, p. 15 .

[2] M.N. Yu, in: R. Behrisch, K.Wittmaack (Eds.), Sputtering by Particle Bombardment III, Springer, Berlin, 1991, p. 91.

[3] J.M. Schroer, T.N. Rhodin, R.C. Braddley, Surf. Sci. 34 (1973) 571.

[4] W.F. van der Weg, P.K. Rol, Nucl. Instr. Meth. 38 (1965) 274.

[5] A. Blandin, A. Nourtier, D.W. Hone, J. Phys. 37 (1976) 396.

[6] G. Blaise, A. Nourtier, Surf. Sci. 90 (1979) 495.

[7] J.K. Norskov, B.I. Lundqvist, Phys. Rev. B 19 (1979) 5661.

[8] R. Brako, D.M. Newns, Surf. Sci. 108 (1981) 253.

[9] N.D. Lang, Phys. Rev. B 27 (1983) 2019.

[10] Z. Šroubek, Phys. Rev. B 25 (1982) 6046.

[11] Z. Šroubek, Nucl. Instr. Meth. 194 (1982) 533.

[12] H.P. Winter, H. Eder, F. Aumayr, Int. J. Mass Spectrosc. 192 (1999) 407.

[13] A. Wucher, Z. Šroubek, Phys. Rev. B 55 (1997) 780.

[14] N.W. Ashcroft, N.D. Mermin, Solid State Physics, Holt, Rinehart \& Winston, New York, 1976.

[15] C.-K. Sun, F. Vallee, L.N. Acioli, E.P. Ippen, J.G. Fujimoto, Phys. Rev. B 50 (1994) 1537.

[16] A.V. Zinoviev, A.V. Lugovskoy, T. Usmanov, Sov. Phys. JETP 71 (1990) 762.

[17] A.V. Lugovskoy, I. Bray, Phys. Rev. B 60 (1999) 3279.

[18] S.S. Johar, D.A. Tompson, Surf. Sci. 90 (1979) 319.

[19] D.A. Tompson, Rad. Eff. 56 (1981) 105. 
[20] H.H. Andersen, H.L. Bay, J. Appl. Phys. 45 (1974) 953.

[21] H.H. Andersen, H.L. Bay, J. Appl. Phys. 46 (1975) 2416.

[22] H.H. Andersen, H.L. Bay, in: R. Behrisch (Ed.), Sputtering by Particle Bombardment I, Springer, Berlin, 1981, p. 194.

[23] P. Sigmund, C. Claussen, J. Appl. Phys. 52 (1981) 990.

[24] S.R. Coon, W.F. Calaway, M.J. Pellin, G.A. Curlec, J.M. White, Nucl. Instr. Meth. B 82 (1993) 329.

[25] Z. Ma, W.F. Calaway, M.J. Pellin, E.I. Von NagyFelsobuki, Nucl. Instr. Meth. B 94 (1994) 197.

[26] Th. Lill, W.F. Calaway, Z. Ma, M.J. Pellin, Surf. Sci. 322 (1995) 361.

[27] A. Wucher, M. Wahl, H. Oechner, Nucl. Instr. Meth. B 82 (1993) 337.

[28] A. Wucher, M. Wahl, Nucl. Instr. Meth. B 115 (1996) 581.

[29] S.F. Belykh, I.S. Bitensky, D. Mullajanov, U.Kh. Rasulev, Nucl. Instr. Meth. B 129 (1997) 451.
[30] S.F. Belykh, U.Kh. Rasulev, A.V. Samartsev, S.V. Verkhoturov, I.V. Veryovkin, Mikrochim. Acta (Suppl. 15) (1998) 379.

[31] S.F. Belykh, U.Kh. Rasulev, A.V. Samartsev, I.V. Veryovkin, Nucl. Instr. Meth. B 136-138 (1998) 773.

[32] S.F. Belykh, B. Habets, U.Kh. Rasulev, A.V. Samartsev, L.V. Stroev, I.V. Veryovkin, Nucl. Instr. Meth. B 164-165 (2000) 809.

[33] P. Sigmund, Phys. Rev. 184 (1969) 383.

[34] J. Lörinčík, Z. Šroubek, H. Eder, F. Aumayr, H.P. Winter, Phys. Rev. B 62 (2000) 16116.

[35] Y. Yamamura, Nucl. Instr. Meth. B 33 (1988) 493.

[36] P. Sigmund, J. Phys. (France) 50-C2 (1989) 175.

[37] V.I. Shulga, P. Sigmund, Nucl. Instr. Meth. B 47 (1990) 236.

[38] V.I. Shulga, P. Sigmund, Nucl. Instr. Meth. 62 (1991) 23.

[39] P. Sigmund, I.S. Bitensky, J. Jensen, Nucl. Instr. Meth. B 112 (1996) 1. 